\title{
Turbulent dissipation in drag reduced flows
}

Bettina Frohnapfel (Karlsruhe Institute of Technology, Germany)

Andrea Cimarelli (Università Politecnica delle Marche, Italy)

Yosuke Hasegawa (The University of Tokyo, Japan)

Maurizio Quadrio (Politecnico di Milano, Italy)

Davide Gatti (Karlsruhe Institute of Technology, Germany)

The question whether turbulent dissipation increases or decreases in turbulent channel flows with turbulent skin friction drag reduction is contradictorily discussed in literature. In order to shed light into this issue we choose to investigate it within the Constant Power Input (CPI) framework, in which the total power fed to the system and thus to total energy dissipation rate is given and constant.

We derive new exact relationships between the Reynolds shear stress (i.e. turbulent skin-friction drag) and the dissipation rates of turbulent and mean kinetic energy, along with all terms in the kinetic energy budgets of turbulent channels. An alternative decomposition of the kinetic energy dissipations is adopted, stemming from an extended Reynolds decomposition, in which the mean velocity is additionally split into a laminar component and a deviation from it. A compact representation of the energy fluxes is introduced to understand their relative importance at different values of the Reynolds number. The particular properties of the laminar and deviation component allow deriving exact relationships that link all the energy fluxes with two wall-normal integrals of the Reynolds shear stresses.

The newly-derived relationships show that drag reduction and turbulent dissipation are linked via the two integrals of the Reynolds shear stresses and a power-based Reynolds number only. This implication of this result is discussed for a number of different flow control scenarios which are realized through DNS of turbulent channel flows under CPI. 